\title{
CONTENCIONES MECÁNICAS EN GERIATRÍA: PROPUESTAS TÉCNICAS Y RECOMENDACIONES DE USO EN EL ÁMBITO SOCIAL.
}

\author{
MECHANICAL RESTRAINTS IN THE ELDERLY: TECHNICAL PROPOSALS AND RECOMMENDATIONS \\ FOR USE IN THE SOCIAL SPHERE.
}

JOAN RODRÍGUEZ DELGADO. Fisioterapeuta del Centre Gerontològic Montsacopa, Olot (Girona). Profesor de la Facultad de Ciencias de la Salud y el Bienestar de la Universidad de Vic (Barcelona).

CORRESPONDENCIA: Centre Gerontològic Montsacopa. C/ Del rengle, 1. 17800, Olot (Girona). Teléfono: 9722602 02. Fax: 9722722 81. Correo electrónico: irodriguez@montsacopa.com

\begin{abstract}
RESUMEN
La revisión bibliográfica de la literatura gerontológica estatal desvela alguna confusión en la utilización de los términos que hacen referencia a las contenciones mecánicas, escasez de diálogo ante los conflictos éticos que sugieren su uso, una notable generalización de las afirmaciones en contra y la ausencia de referencias positivas a pesar de que como muestran algunos autores su prevalencia es de las más elevadas del mundo. En este trabajo se exponen algunas propuestas técnicas relativas a la definición, el empleo de los términos y el uso de las contenciones mecánicas en el ámbito social como anteponer el diálogo ético a las argumentaciones basadas en la prevalencia, definirlas en función de su intencionalidad, consensuar una clasificación de los diferentes métodos de contención, identificar los tipos y los niveles de riesgo e intervenir específicamente y en función de los mismos. Se añaden al final recomendaciones relativas al riesgo, al proceso de decisión, la prescripción y al proceso de retirada.
\end{abstract}

Palabras clave: restricción física, ancianos, conflicto ético, ámbito social.

\begin{abstract}
MECHANICAL RESTRAINTS IN THE ELDERLY: TECHNICAL PROPOSALS AND RECOMMENDATIONS FOR USE IN THE SOCIAL SPHERE.
\end{abstract}

\section{ABSTRACT}

The bibliographical revision of national gerontological literature reveals some confusion in the use of terms that refer to mechanical contentions, lack of dialogue with regards to ethical conflicts that suggest their use, a significant generalization of the claims against and the absence of positive references despite its high prevalence as shown by some authors. This paper presents some technical proposals on the definition, the use of terms and the use of mechanical contentions in the social sphere such as putting the ethical dialogue before the argumentation based on the prevalence, define them in terms of their intent, agree on a classification of the different contention methods, identify the types and levels of risk and intervene specifically and in terms thereof. Finally, recommendations are added with regards to risks, the decision process, prescription and the withdrawal process.

Keywords: physical restraint, elderly, ethical conflicts, social sphere. 


\section{Introducción}

El Diccionario de la Real Academia Española define "contener" como el hecho de "sujetar el movimiento de un cuerpo"1 y al verbo "sujetar" le da una connotación física con matices relacionados con la dominación y el poder². El Diccionari de la Llengua Catalana describe el término "contener" como el hecho de impedir que una persona o cosa salga de sus límites o impedir que traspase un límite, refiriéndose no sólo a algo físico sino también emocional ${ }^{3}$. Por tanto "sujetar" y "contener" no son lo mismo aunque se usen como sinónimos. Hay muchos tipos de contención y no sólo mecánicas, como veremos más adelante, pero en el ámbito en el que nos movemos la definición más aceptada es la que se refiere a la utilización de un dispositivo físico y/o mecánico para restringir los movimientos de una parte del cuerpo o su totalidad con el fin de prevenir aquellas actividades físicas que puedan poner en situación de riesgo 0 en peligro de lesión a la persona enferma o a otras personas de su entorno ${ }^{4}$. La mayoría de las definiciones que aparecen en la literatura consultada son muy parecidas a la citada o contienen algunas pequeñas variaciones que señalan la proximidad al cuerpo de los dispositivos y la dificultad para retirarlos ${ }^{5-11}$. El uso de los términos entre la comunidad científica se decanta mayoritariamente entre "sujeción" y "restricción"5-10,12-17 para referirse en la práctica a las muñequeras, cinturones de sujeción abdominal o pélvica, chalecos de sujeción torácica 0 integral y los cinturones de cama. No queda claro si otros dispositivos, tales como las barandillas de la cama, también pueden considerarse como tales, ya que unos autores la consideran una contención clara ${ }^{7,9,15,16}$, Gobert et al. especifican que es un tipo de restricción añadida al mobiliario ${ }^{5}$, Burgueño deja entrever que puede considerarse una contención si existe intencionalidad de levantarse de la cama ${ }^{18}$, y otros autores y organismos no la consideran, ${ }^{4,21}$. Los sobrenombres "física" o "mecánica" constituyen otra de las posibles confusiones por ser utilizados como sinónimos entre los profesionales de la geriatría para referirse a los dispositivos ${ }^{4,19}$, pero no así en otros ámbitos de la intervención social tales como infancia, adolescencia y discapacidad intelectual donde la "contención física" es la realizada cuerpo a cuerpo entre el cuidador y la persona atendida, sin mediación de ningún dispositivo.

Un elemento de conflicto en que se basan algunas campañas contrarias al uso de sujeciones es el argumento de que en España la prevalencia es de las más elevadas del mundo, concretamente un $39,6 \%$, basándose en una publicación de Ljungren $\mathrm{G}$. et al. del año $19977-9,11,14,18$, cuando en la actualidad no hay suficiente evidencia ni estudios que clarifiquen la situación estadística real, excepto un reciente e interesante trabajo de campo realizado por el Servicio de Inspección y Registro del Departament de Benestar Social i Família de la Generalitat de Catalunya que dice que de una población de 8772 personas mayores distribuidas entre 164 residencias del territorio, la prevalencia general es del $21,5 \%$ y del $41,2 \%$ si tienen demencia 22 .

Otro más y de los más serios argumentos utilizados en algunos documentos de relevancia es decir que atentan contra las bases del cuidado y que las restricciones y sus alternativas limitadoras son una "forma clara de maltrato" o incluso "un acto de violencia en sí mismo", a mi entender confundiendo el dispositivo con el uso que se le da ${ }^{12,18}$. A pesar de ello, y excepto la enumeración de algunos principios éticos afectados como la autonomía, la libertad y la dignidad, de nuestra revisión se destaca la escasez de referencias éticas sobre el uso de las contenciones ${ }^{4,}$, 12, 22 y la ausencia de referencias positivas de uso a pesar de la citada y elevada prevalencia, la notable generalización de las afirmaciones en contra de las contenciones y la ausencia de supuestos y circunstancias específicas de uso. Faltan elementos de juicio y referencias donde se aborden de manera consistente tanto los conflictos técnicos como éticos por el uso de contenciones.

Por último, el uso de los dispositivos es una de las principales indicaciones para la prevención de algunas caídas, tanto a nivel institucional $7,8,12,14-16,20$, como familiar ${ }^{9}$ y simultáneamente están contraindicados por los efectos adversos para la salud, tanto físicos como psíquicos, o incluso la muerte, por su uso indebido tal como muestran muchos de los trabajos consultados ${ }^{6}$ 12,14-16,18 
Ante la escasez de referencias éticas, la ausencia de referencias positivas, de supuestos y circunstancias específicas de uso de las contenciones mecánicas y ante los conflictos y ambigüedades surgidas de nuestra revisión, aportamos algunas propuestas técnicas y algunas recomendaciones para facilitar en lo posible el uso de las medidas que nos ocupan entre las personas mayores del ámbito social.

\section{Propuestas técnicas}

Anteponer el diálogo ético a los argumentos basados en la prevalencia

En el ámbito social todas las intervenciones técnicas y profesionales están o deberían estar al servicio de las personas mayores, los principios éticos fundamentales y las virtudes del cuidar. La dignidad, entendida como aquello propiamente humano y merecedor de respeto, se puede poner en entredicho ante algunas situaciones de riesgo para nuestros usuarios durante la aplicación de algunas medidas para contrarrestarlas y puede mezclarse de cuestiones más relativas a la cultura o la estética que al principio fundamental. Por ejemplo, con tal de impedir la bipedestación en algunos ancianos con riesgo grave o inminente de caer y con tal que no lleven un "cinturón de seguridad" que contabilice en la prevalencia se le puede ubicar en un confortable sillón bajo, consiguiendo el mismo efecto restrictivo. En este caso aunque no se utiliza un dispositivo mecánico y estéticamente está más aceptado el sillón que la contención, debemos preguntarnos si igualmente se están vulnerando los principios fundamentales. De igual forma pasa con el riego de caídas, ante las cuales algunos familiares y profesionales les es más difícil aceptar los discursos amparados en la ética que sostener las poderosas emociones, especialmente miedo, que se despiertan a favor de la contención, cuando ven a su familiar o usuario con un impactante hematoma o corte después de haber caído. Ante éstos casos ¿qué prevalece más, la libertad de caer o la de prevenir y contener el riesgo? Ante la posibilidad del uso de contenciones propongo plantearnos si como mínimo su aplicación es menos maleficente, en términos éticos, que su no aplicación.

Otro planteamiento a este nivel que aparece en la literatura es sobre la libertad y la autonomía. La libertad, entendida como el hecho de poder escoger entre más de una alternativa sin coacción y llevarla a cabo y la autonomía entendida como la capacidad de decidir, merecen un escrupuloso trato. Ante las personas que se caen repetidamente y deciden aceptar las consecuencias no suelen existir conflictos y por tanto no deberían usarse los dispositivos de contención mecánica si preservan su libertad y autonomía. No es así cuando la persona no goza de estos principios. Pongo como ejemplo a ancianos dependientes y con demencias muy avanzadas, con índices de Barthel inferior a 19/100, escala de deterioro global de Reisberg de $7 / 7$ y test de Tinetti de 1 a 3/28, ante los cuales creemos que un cinturón abdominal o pélvico son más una medida de seguridad para facilitar la sedestación y vida social que una medida de restricción. Al respecto, no estamos de acuerdo con los autores que asocian el maltrato y la violencia al dispositivo en sí mismo ${ }^{12,18}$, excepto si se usan por dominación, abuso de poder o conveniencia. Entendemos que es más la intención de uso que el material lo que determina el conflicto ético.

Si aceptamos que la ética y el diálogo son la base de la asistencia, es difícil verle el sentido a la "tolerancia cero" o las campañas de retirada basadas en la prevalencia, y más aún cuando no disponemos de demasiados datos excepto los ya citados. Decir que una residencia tiene una prevalencia del $20 \%$ de contenciones o que un centro está libre de ellas no aporta más que un dato estadístico competitivo 0 incluso vacío de contenido si no se aviene a la ética asistencial y a la realidad. Una propuesta en este sentido es correlacionar la prevalencia con el perfil de los usuarios, los datos de riesgo, especialmente el de caídas y los programas de prevención de las mismas, y la introducción del diálogo ético en los equipos asistenciales, supervisados por especialistas 0 entidades autónomas. 
Definir las contenciones mecánicas en función de su intencionalidad

El término genérico "contención" hace referencia a una acción como el hecho de poner un límite a la persona, no desde la dominación o el poder sino como algo bien intencionado más beneficioso que perjudicial con la intención, valga la redundancia, de impedir que traspase un límite físico y/o emocional. En base a esto definir las contenciones mecánicas en función de los dispositivos crea ambigüedades tanto técnicas como éticas. El ejemplo de la barandilla de la cama que según Burgueño ${ }^{18}$ puede considerarse una contención si existe "intencionalidad" de levantarse o no, es claro. ¿Por qué no hacer hincapié en la intencionalidad más que en los dispositivos? Centrarnos en que las contenciones mecánicas son el chaleco, el cinturón u otros y que éstos son la cosa non grata a erradicar puede hacer surgir otros procedimientos, como el citado del sillón confortable o una mesa incorporada a la silla de ruedas que ejercen el mismo efecto restrictivo que los dispositivos tradicionales pero podríamos vulnerar igualmente los principios fundamentales. Proponemos ampliar el concepto de las contenciones mecánicas a cualquier procedimiento, 0 dispositivo técnico, mobiliario o textil en contacto o muy cercano a la persona cuya intención sea la de restringir, limitar 0 dificultar su movilidad de una 0 diversas partes de su cuerpo.

Consensuar una clasificación de contenciones en el ámbito social

Las contenciones son procedimientos habituales entre los colectivos que reciben intervención social: personas mayores, infancia y adolescencia, personas con enfermedades mentales y personas con discapacidad intelectual. Los más conocidos y utilizados son el time out o separación de una persona de un entorno que altera o puede alterar la conducta, la contención espacial o ubicación en salas o espacios amplios controlados, la contención verbal que se realiza mediante el uso de la palabra, la contención física, que es la que se realiza cuerpo a cuerpo entre el cuidador y el usuario sin mediación de dispositivos, la contención farmacológica, también llamada contención química y la contención mecánica. Todos estos procedimientos también son utilizados en el ámbito de la geriatría pero excepto la contención farmacológica no suelen ser contemplados, 0 al menos en la literatura consultada. Proponemos aprovechar la riqueza de todos los ámbitos sociales y consensuar una clasificación donde además de las contenciones mecánicas, tengamos en cuenta todas las demás.

Si nos centramos en las contenciones mecánicas, basándonos en la definición propuesta, podemos considerar como tales a los conocidos y ya citados dispositivos de sujeción, sean homologados o no, tipo cinturón abdominal, tanto de cama como de silla, cinturón pélvico, chaleco de sujeción torácica o integral, muñequeras, tobilleras o cualquier dispositivo parecido que restrinja la movilidad de alguna parte del cuerpo 0 su totalidad. Pero también se pueden incluir las barandillas de cama, las mesas incorporadas a la silla o diferentes dispositivos textiles como vestidos adaptados 0 sábanas ajustables a la cama habitualmente con cremallera $u$ otros tipos de cierre que sólo dejan salir una o diversas partes del cuerpo, si su intención es limitar o restringir su movilidad. Hay algunos procedimientos que también pueden considerarse como métodos de contención mecánica como es el caso de las butacas o sillones bajos, algunas sillas adaptadas y butacas reclinables, las sillas de ruedas frenadas delante de una mesa o la disposición concreta del mobiliario si su intención coincide con la ya citada.

Identificar los riesgos e intervenir en función de los mismos

En la literatura consultada hay acuerdo en identificar de manera parecida las principales indicaciones de uso de las contenciones mecánicas en geriatría 4,7,8,12,14,16,20: disminuir o prevenir el riesgo de caídas y lesiones, controlar las conductas de riesgo para la seguridad propia o terceros, proteger la manipulación de equipos médicos y mantener la posición anatómica. Hay autores $^{8,12}$ que además enumeran otros argumentos para su utilización: prevenir el estado de confusión y 
agitación, eliminar las conductas de vagabundeo, impedir movimientos repetitivos, vencer las resistencias a tratamientos 0 alimentación, controlar el riesgo ante las alteraciones del sueño 0 por deseo de los familiares.

Observamos que excepto para las caídas, no quedan bien identificadas ante qué otras conductas de riesgo están indicadas las contenciones ni existe una correlación de uso para cada tipo de riesgo ni circunstancias específicas de uso. En la intervención social en las persones mayores podemos identificar tres tipos generales de riesgo: caídas, trastornos de la conducta y las salidas no controladas de los ancianos afectados por desorientación o demencia. Cada uno de estos riesgos generales se puede desgranar en riesgos específicos: las caídas pueden ocurrir desde el decúbito en la cama, la sedestación o durante la bipedestación, transferencias o marcha; los trastornos de conducta pueden suponer un riesgo para sí mismo como la ansiedad y la angustia o para terceros, como en el caso de la agresividad; y algunos de los riesgos específicos de las salidas no controladas son la desorientación y la pérdida en el exterior.

Una vez identificados los riesgos, la intervención debe ser coherente y correlativa tanto en su aplicación como en su intensidad. En la intervención en las personas mayores, en el ámbito social son raros los trastornos psiquiátricos tan severos que requieran de contenciones mecánicas y podemos hacer uso de otros métodos de contención ya sea time out, verbal, física (cuerpo a cuerpo), química o espacial. Las salidas no controladas al exterior tampoco son una indicación para las contenciones mecánicas, aunque disponemos de un interesante estudio de Fariña-López et al. ${ }^{9}$ que en el ámbito familiar este motivo ocupa un $37,2 \%$. En este caso abogamos por otro tipo de intervenciones que potencien el paseo y la salida controlada, el uso de dispositivos con localizador GPS o la contención espacial como último recurso. La única indicación que nos sugieren las contenciones mecánicas en nuestro ámbito es la prevención de algunas caídas.
Identificar el nivel de riesgo de caídas e intervenir específicamente

Las caídas son la primera causa de lesiones, incapacidad y pérdida de autonomía ${ }^{23-25}$ y hay acuerdo en el elevado coste por la gravedad de las consecuencias físicas, funcionales, psicológicas, sociales y económicas que suponen y por ser un problema de salud pública de primera magnitud ${ }^{25-27}$. Nuestros datos de seguimiento revelan que en los dos últimos años hay una coincidencia entre la disminución de la prevalencia de las contenciones mecánicas con el aumento de la de las caídas y aunque no podemos hacer una correlación directa causa-efecto sí que es observable en la práctica que algunas personas con demencia avanzada que caen reiteradamente responden bien a la contención mecánica respecto a otras alternativas, reduciéndose drásticamente el riesgo y el número de caídas. El riesgo de caer, según expresan algunos instrumentos como el test de Dowton, puede ser leve, moderado o severo, por lo que asignar un dispositivo de sujeción a las primeras de cambio lo consideramos tan inapropiado como renunciar a ponerlo cuando hay un riesgo de daño severo e inmediato, en contra de lo que escribe Tideiksaar en su excelente libro ${ }^{20}$, si no hemos sido capaces de prevenirlo de otra manera.

Las caídas son la consecuencia de factores tanto extrínsecos como intrínsecos que deben ser bien diagnosticados y tratados coherentemente. La detección y la intervención basada en los factores de riesgo constituyen el primer nivel de intervención y sólo en el caso de que tales estrategias proactivas y preventivas no funcionen debemos plantearnos las intervenciones contenedoras de progresiva intensidad.

\section{Recomendaciones de uso}

En base a todo lo descrito en este documento proponemos, entre otras, las siguientes recomendaciones sobre el uso de las contenciones mecánicas en las personas mayores del ámbito social: 
Respecto al riesgo

- Identificar los tipos y niveles de riesgo que puedan sufrir los usuarios.

- Identificar los riesgos que pueden sugerir el uso de contenciones mecánicas.

- Revisar y tratar los factores de riesgo de caídas tanto intrínsecos como extrínsecos.

- Promocionar las actividades preventivas, cuidadoras, rehabilitadoras y las medidas de seguridad: trato humano, agradable $y$ respetuoso basado en los principios fundamentales, exhaustivo control de los psicofármacos y la polifarmacia, fisioterapia y rehabilitación de la marcha y el equilibrio, actividades físicas destinadas a la prevención de caídas, adaptación del entorno arquitectónico y mobiliario.

\section{En el proceso de decisión}

- Respetar la autonomía y la libertad de las personas que asumen sus propios riesgos.

- En las personas sin autonomía ni competencia para tomar decisiones, consensuar siempre con la familia o tutor y el equipo profesional.

- Las decisiones con componente ético deben ser consensuadas en equipo y que no recaigan exclusivamente en el facultativo aunque así esté regulado por las leyes $28-29$.

\section{Antes de la prescripción}

- Disponer del consentimiento informado del usuario, familiar o tutor de referencia ${ }^{30,31}$.

- Preguntarse si el riesgo es inminente, si no hay alternativas, si ya se ha probado todo, si es la última opción, si es menos maleficente que el riesgo, si estamos vulnerando algún principio ético, si tenemos el consentimiento de todos, si hemos previsto las consecuencias de su aplicación, si la medida de contención es adecuada al tipo y al nivel de riesgo.
Durante el periodo de prescripción

- Mantener el consenso de todas las partes.

- Controlar exhaustivamente las consecuencias por el uso de la contención.

- Establecer programas de intervención: movilidad, paseo, hidratación, rondas al WC, control de la piel, control de la incontinencia, cambios en el entorno, promocionar la visita de familiares, amigos, profesionales 0 voluntarios.

- Seguir manteniendo todas las actividades estimuladoras, lúdico-recreativas 0 de mantenimiento.

En el proceso de retirada

- Preguntarse si sigue siendo menos maleficente la contención que la retirada y si todas las partes están de acuerdo.

\section{En la gestión del proceso}

- Que la prescripción sea consensuada por el equipo.

- Disponer de la información y formación suficiente, tanto en ética, técnica y uso de las contenciones como sensibilización ante su uso. Recomendamos la realización de seminarios y talleres de sensibilización ${ }^{32}$.

- Asegurar que el trabajo es en equipo y sigue los estándares de calidad.

- Control continuado y revisión mensual de los indicadores del proceso de contención relacionado con otros procesos como el de riesgos, caídas y movilidad.

- Fomentar la participación y las actividades educativas para familiares y profesionales. 


\section{CONFLICTO DE INTERESES}

El autor declara no tener ningún conflicto de intereses.

\section{AGRADECIMIENTOS}

Al Dr. Salvador Vilar i Subiràs, director del Centre Gerontològic Montsacopa de Olot (Girona) por la revisión del documento y sus aportaciones éticas. Al Dr. Xavier Cardona Iguacen, a la Sra. M ${ }^{a}$ Mercè Vidal Puig, a la Dra. Laura Vergés Planagumà, al Sr. Pep Bové Rivé y a la Sra. Toñi Segura Baeza, miembros del Grupo de trabajo sobre contenciones del Comité de Ética de Servicios Sociales de la Generalitat de Catalunya. A Kerstin Orth educadora social especializada en problemas psicosociales de la adolescencia.

\section{BIBLIOGRAFIA}

1. Diccionario de la Real Academia de la Lengua Española. Disponible en: http://lema.rae.es/drae/?val=contenci\%C3\%B3n. [Consulta: 02/01/2013].

2. Diccionario de la Real Academia de la Lengua Española. Disponible en: http://lema.rae.es/drae/?val=sujetar. [Consulta: 18/8/2011]

3. Diccionari de la llengua catalana. Disponible en: http://dlc.iec.cat/results.asp?txtEntrada=contenir\&operEntrada=0. [Consulta: 02/01/2013].

4. Col-legi Oficial d'Infermeria de Barcelona, comissió deontològica. Consideracions davant les contencions físiques i/o mecàniques: aspectos ètics $i$ legals. Barcelona. 2006.2 Disponible en: http://www.coib.org/uploadsBO/noticia/Documents/DOC\%20CONTENCIONS_DE F.PDF [Consulta: 18/8/2011]

5. Gobert M, d'Hoore W, Mora-Fernández J, et al. Adecuación de las restricciones físicas y farmacológicas en los ancianos institucionalizados: estudio comparativo en Québec y la Suiza Romanda, con implicaciones en nuestro medio. Rev Esp Geriatr Gerontol. 2005;40(1):7-17.

6. Zunzunegui M.V. Restricciones físicas y farmacológicas de las personas mayores que viven en instituciones. Rev Esp Geriatr Gerontol. 2005;40(1):4-6.

7. Galán $\mathrm{CM}^{\mathrm{a}}$, Trinidad $\mathrm{D}$, Ramos $\mathrm{P}$, et al. Uso de sujeciones físicas en una población anciana ingresada en residencias públicas. Rev Esp Geriatr Gerontol. 2008:43(4):208-13
8. Alarcón T. Uso de restricción física en el anciano en el siglo XXI ¿Necesidad o falta de formación? Rev Esp Geriatr Gerontol. 2001;36(1):46-50

9. Fariña-López E, Estévez-Guerra G, Núñez E, et al. Estudio descriptivo sobre la actitud de la familia ante el uso de restricciones físicas en mayores: resultados preliminares. Rev Esp Geriatr Gerontol. 2008;43:201-7.

10. Del Río $M$, Gótor $P$, Cuevas $P$, et al. Restricciones físicas en pacientes ancianos hospitalizados. Perfil de los pacientes y motivos de su uso. Disponible en: http:/semerwp.saluddigital.net/wpcontent/uploads/2010/09/restriccionesresidencias.pdf [Consulta: 18/8/2011]

11. Confederación Española de Organizaciones de Mayores (CEOMA). Guía para personas mayores y familiares: uso de sujeciones físicas y químicas con personas mayores y enfermos de Alzheimer. Programa "Desatar al anciano y al enfermo de Alzheimer". Disponible en http://www.ceoma.org/alzheimer/pdfs/guia_para_personas_mayores.pdf. [Consulta: 17/8/2011].

12. Sociedad Española de Geriatría y Gerontología. Hacia una cultura sin restricciones: las restricciones físicas en ancianos institucionalizados. Documento técnico, núm. 3. Julio 2003.

13. Orbegozo, A. Experiencias en relación con el maltrato y el abordaje desde las instituciones y entidades in De la Cuesta, J.L. El maltrato de personas mayores. Hurkoa Fundazioa. Instituto Vasco de Criminología. Disponible en: http://www.ehu.es/p200-

content/en/contenidos/informacion/ivckei_jose_luis_delacuesta/es_joseluis/adjunt os/Libro_Maltrato_web.pdf. [Consulta 04/02/2013]

14. Alarcón T. El uso adecuado de la restricción física en el anciano: una preocupación creciente. Rev Esp Geriatr Gerontol. 2008;43(4):197-8.

15. Fariña-López E, Camacho A, Estévez-Guerra E, et al. Accidentes asociados al uso de restricciones físicas en ancianos con trastornos cognitivos: estudio de tres casos. Rev Esp Geriatr Gerontol. 2009;44(5):262-5.

16. Fariña-López E. Problemas de seguridad relacionados con la aplicación de dispositivos de restricción física en personas mayores. Rev Esp Geriatr Gerontol. 2011:46(1):36-42.

17. Suen LKP, Lai Cky, Wong TKS, et al. Restricciones físicas en cuidados de larga duración: actitudes, experiencia y conocimiento mejoran su uso. Rev Enferm Clin. 2006;16(6):345-7

18. Burgueño A. Atar para cuidar: uso de sujeciones físicas y químicas en personas mayores dependientes que reciben cuidados prolongados. 2008. Disponible en: http://www.navarra.es/NR/rdonlyres/5E315864-34F9-4236-9319931E610416DB/107054 /usodesujecionesenpersonasmayores1.pdf [Consulta: $17 / 8 / 2011]$

19. Joint Commission International. Joint Commission International Standards for the Care Continuum. First Edition. Joint Commission on Accreditation of Healthcare Organizations. 2003.

20. Tideiksaar R. Caídas en ancianos. Ed. Masson. Barcelona. 2005.

21. Departament d'Acció Social i Ciutadania de la Generalitat de Catalunya. Guia per a l'aplicació dels indicadors i procesos per a la millora contínua de la qualitat asistencial 2010 Obtenido 
http://www20.gencat.cat/docs/dasc/01Departament/02Funcionsiestructura/Millora _qualitat_ICASS/Eines_suport_i_publicacions/Publicacions/aplicacio_indicadors_ processos/guia_definitiva.pdf.

22. Servei d'inspecció i Registre. Ús racional de les contencions fisiques. Estudi de la incidència de l'assessorament de la inspecció de serveis socials en residències de gent gran. Generalitat de Catalunya. Departament de Benestar Social i Familia. Barcelona, 2011.

23. García M, Villena A, Del Campo $\mathrm{JM}^{\mathrm{a}}$, et al. Factores de riesgo de caídas en ancianos. Revista CLiN MED FAM 2007; 2 (1): 20-24. Disponible en: http://redalyc.org/src/inicio/ArtPdfRed.jsp?iCve=169617650005.

24. André da Silva Z, Gómez A, Sobral M. Epidemiología de caídas de ancianos en España. Una revisión sistemática. Rev Esp Salud Pública 2008, Vol.82,N01.

25. Neira M, Rodríguez L. Caídas repetidas en el medio residencial. Rev Esp Geriatr Gerontol. 2006; 41 (4):201-6.

26. Villar San Pio T, Mesa Lampré MP, Esteban Gimeno AB, Sanjoaquín Romero AC, Fernández Ariu E. Alteraciones de la marcha, inestabilidad y caídas. En Tratado de geriatría para residentes. Madrid. Ed. Sociedad Española de Geriatria y Gerontología. Madrid. 2006. p 199-209.

27. Rapado A, Guillén F. Osteoporosis y caídas en el anciano. Ed. Fahoemo. 1994. Barcelona.

28. Diari Oficial de la Generalitat de Catalunya. DOGC núm. 4990 - 18/10/2007. Llei 12/2007 de Serveis Socials. Disponible a: http://www.gencat.cat/diari/4990/07284064.htm. [Consulta: 18/8/2011].

29. Decreto Foral de Navarra. Disponible en: http://benasque.aragob.es:443/cgibin/CONV/BRSCGI?CMD=VEROBJ\&MLKOB=624330572625. [Consulta: 04/02/2013].

30. Diari Oficial de la Generalitat de Catalunya. Llei $21 / 2000$, de 29 de desembre, sobre els drets d'informació concernent la salut i l'autonomia del pacient, i la documentació clínica. Disponible en: http://www.gencat.cat/salut/depsalut/pdf/eu22.pdf. [Consulta: 18/8/2011].

31. Generalitat de Catalunya. Departament de Sanitat i Seguretat Social. Comitè de Bioètica de Catalunya. Guia de recomanacions sobre el Consentiment informat.Barcelona. $2003 . \quad$ Disponible http://www.gencat.cat/salut/depsalut/pdf/consentiment.pdf. [Consulta: 18/8/2011].

32. Rodríguez Delgado J. Contencions mecàniques: diàleg a partir de les sensacions del professional. Resultats i conclusions del taller de sensibilització sobre l'ús de les contencions físiques. Olot. 2011. Disponible en: http://solofisio.files.wordpress.com/2011/10/taller-de-sensibilitzacic3b3-sobreIc3bas-de-les-contencions-resultats-i-conclusions.pdf. 